\section{MS5-P33 Structure-functional relationships of a novel haloalkane dehalogenase with two halide-binding sites}

Tatyana Prudnikova ${ }^{1,2}$, Radka Chaloupkova ${ }^{3}$, Pavlina Rezacova $^{4,5}$, Jiri Damborsky ${ }^{3}$, Ivana Kuta-Smatanova ${ }^{1,2}$

1. Faculty of Science, University of South Bohemia in Ceske Budejovice, Ceske Budejovice, Czech Republic

2. Institute of Nanobiology and Structural Biology, Academy of Sciences of the Czech Republic, Nove Hrady, Czech Republic

3. Loschmidt Laboratories, Department of Experimental Biology and Research Centre for Toxic Compounds in the Environment RECETOX, Masaryk University, Brno, Czech Republic

4. Institute of Molecular Genetics, Academy of Sciences of the Czech Republic, Prague, Czech Republic

5. Institute of Organic Chemistry and Biochemistry, Academy of Sciences of the Czech Republic, Prague, Czech Republic

email: talianensis@gmail.com

Haloalkane dehalogenases (EC 3.8.1.5) are bacterial enzymes cleaving a carbon-halogen bond by a hydrolytic mechanism in a broad range of halogenated aliphatic compounds [1]. Novel haloalkane dehalogenase DbeA from Bradyrhizobium elkanii USDA94 was structurally and biochemically characterized in this study. The crystal structure of DbeA was solved to $2.2 \AA$ resolution and revealed the presence of two halide-binding sites. The first chloride-binding site was located in the active site in between two halide-stabilizing residues. The second chloride-binding site was buried deeply in the protein core and was approximately $10 \AA$ from the first binding site. This second halide-binding site is unique to DbeA and has not been previously reported in any other crystal structure of this enzyme family. To elucidate the role of the second halide-binding site in enzyme functionality, a two-point mutant (I44L+Q102H) lacking this site was constructed, purified and biochemically characterized. Its comparison with the wild type enzyme revealed that elimination of the second halide-binding site decreased stability of the enzyme in the presence of chloride salt and decreased its catalytic activity by an order of magnitude. Moreover, the two-point substitution resulted in a shift of the substrate-specificity class, which is the first time this has been demonstrated for the haloalkane dehalogenase enzyme family. Changes in the catalytic activity of the variant were attributed to deceleration of the rate-limiting hydrolytic step, mediated by lower basicity of the catalytic histidine [2].

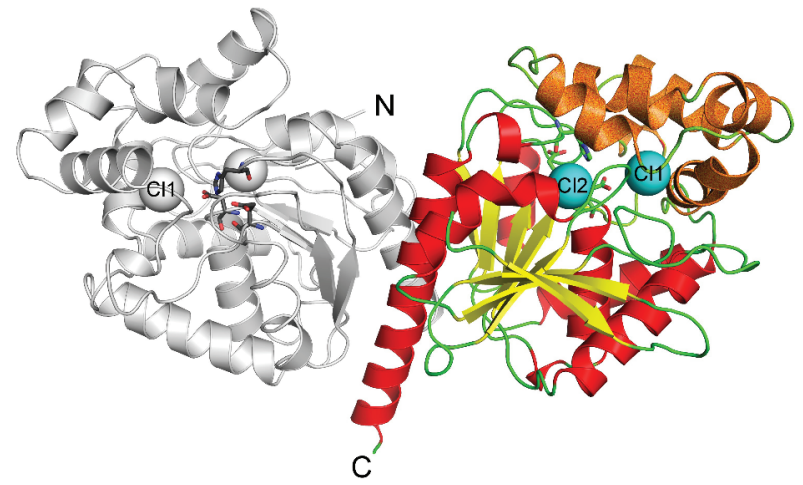

Figure 1. Overall structure of the haloalkane dehalogenase DbeA.
MS5-P34 Structure-functional studies of selected haloalkane dehalogenases

Ivana Kuta Smatanova ${ }^{1,2}$

1. Faculty of Science, University of South Bohemia in Ceske Budejovice, Branisovska 31, 37005 Ceske Budejovice, Czech Republic

2. Institute of Nanobiology and Structural Biology, Academy of Sciences of the Czech Republic, 14220 Prague, Czech Republic

email: ivanaks@seznam.cz

X-ray crystallography is the major technique to get the structure of biological macromolecules at atomic resolution. Protein structures are central to understand the detailed mechanisms of biological processes and to discover novel therapeutics, biosensors, etc. using a structure-based approach. Several non-membrane and membrane protein complexes, which are important for living on the earth, have been crystallized using different crystallization techniques such as standard, advanced and alternative methods.

At this time several haloalkane dehalogenases, their mutant variants and complexes with substrates are systematically studied in our laboratory. Developed methods, obtained crystallization and crystallographic data were compiled and results were published in scientific journals [1]. This presentation will be focused on description and comparison of crystallization conditions and crystallographic results of selected proteins such as DhaA from Rhodococcus rhodochrous NCIMB 13064, DbeA of Bradyrhizobium elkani USDA94, LinB of Sphingobium japonicum UT26, DpcA from Psychrobacter cryohalolentis K5, and DmxA from Marynobacter sp ELB 17.

This research is supported by the GACR (P207/12/0775).

1. Chaloupkova R, et al., Acta Crystallogr. D Biol. Crystallogr. 70, 1884-1897 (2014)

Keywords: haloalkane dehalogenases, crystallization, structure

Keywords: Haloalkane dehalogenase, halide-binding site, 